\title{
PROGRAMA “CRECIENDO” (CREANDO COMPETENCIAS DE INTELIGENCIA EMO- CIONAL EN NUEVOS DOCENTES): EVIDENCIAS PRELIMINARES Y SU UTILIDAD PERCIBIDA EN LA FORMACIÓN INICIAL DEL PROFESORADO DE SECUNDARIA
}

\author{
Natalio Extremera, Sergio Mérida-López, Lourdes Rey y María Ángeles Peláez-Fernández \\ Universidad de Málaga, España
}

\section{Resumen}

El profesorado novel es cada vez más consciente de la necesidad de desarrollar sus habilidades emocionales para ser profesionales competentes en su futura labor docente. Sin embargo, la formación en educación emocional recibida por el profesorado novel en los Másteres Oficiales de Enseñanza del Profesorado de Secundaria todavía es escasa. La presente investigación presenta una experiencia piloto llevada a cabo en el Máster en Profesorado de Secundaria de la Universidad de Málaga con un grupo de 14 docentes noveles, en la cual se evaluaron los niveles de inteligencia emocional antes y después del taller, su evaluación percibida sobre diferentes indicadores a corto $\mathrm{y}$ largo plazo como resultado de la formación, así como la valoración de aspectos positivos y negativos, grado de cumplimiento de las expectativas y aprendizajes adquiridos. A pesar de la brevedad del programa, los resultados han mostrado que los docentes noveles participantes incrementaron algunas habilidades emocionales. Igualmente, se subraya la utilidad y aplicabilidad percibida del programa para gestionar los conflictos en el aula, así como diversos aspectos positivos y negativos de esta experiencia piloto. Se sugieren implicaciones prácticas y futuras líneas de trabajo.

Palabras clave: Inteligencia emocional, Formación, Profesorado novel, Utilidad percibida del entrenamiento
Abstract

Pre-service teachers are increasingly aware of the need to develop their teacher emotional skills to become more competent professionals in their future teaching work. However, training in emotional education received by new teachers in Spanish Master's degrees in Teacher Training for Secondary Education is still scarce. This study presents a pilot experience carried out in the Master's degree of Secondary Education of the University of Malaga with a group of 14 pre-service teachers, in which the levels of emotional intelligence were evaluated before and after the workshop, as well as their perceived evaluation on different short and long-term indicators as a result of the training, judgements of positive and negative aspects, expectations fulfilment and acquired knowledge regarding the program. Despite the brevity of the program, the results have shown that participating pre-service teachers increased some emotional skills. Likewise, the usefulness and perceived applicability of the program to manage conflicts in the classroom as well as some positive and negative aspects of this pilot experience are highlighted. Practical implications and future lines of work are suggested.

Keywords: Emotional intelligence, Training, Pre-service teachers, Perceived training utility

Correspondencia.

Natalio Extremera

nextremera@uma.es

Departamento de Psicología Social, Trabajo Social, Antropología Social

y Estudios de Asia Oriental

Universidad de Málaga, Málaga, España 


\section{Introducción}

La enseñanza se ha convertido en una tarea profesional altamente demandante (Montero, 2006), poniendo de relieve el papel que el docente, así como sus niveles de bienestar y sus competencias sociales y emocionales tienen en los procesos de enseñanza y de relaciones interpersonales (Jennings et al., 2011). Como consecuencia, en los últimos años se ha subrayado la necesidad de proporcionar una formación en competencias socio-emocionales a los docentes como forma de proporcionar herramientas educativas y prevenir y/o manejar el estrés y los conflictos diarios de su profesión (Vesely-Maillefer y Saklofske, 2018).

Diferentes trabajos han puesto de manifiesto que los docentes que no pueden manejar de forma efectiva los conflictos en el aula, generándoles estrés y ansiedad, informan de un deterioro en el clima emocional en el aula y sus estudiantes presentan menores niveles de rendimiento y actitudes académicas más negativas (Oberle y Schonert-Reichl, 2017). Además, debido al deterioro en el clima del aula, se llega a producir un contagio emocional entre el profesorado y alumnado, incrementándose los niveles de estrés laboral y burnout en los docentes (Oberle y Schonert-Reichl, 2016). Como consecuencia, los docentes se encuentran más frecuentemente al límite de sus recursos y energía, intentando mantener el control y manejar las clases con un mayor uso de estrategias punitivas, que no promueven un clima emocional adecuado y que podrían reforzar un círculo vicioso de disrupción (Alvarez, 2007).

La Inteligencia Emocional (IE) es un recurso indispensable en las nuevas generaciones de profesionales docentes (Vesely-Maillefer y Saklofske, 2018). En este sentido, las habilidades emocionales de los futuros docentes y su desarrollo podrían jugar un papel clave para desarrollar y mantener un clima de clase positivo y prosocial que podría facilitar una relación profesor-alumno más satisfactoria, favoreciendo el desempeño docente y el aprendizaje del alumnado (Extremera et al., 2019a; Jennings y Greenberg, 2009). Asimismo, la IE docente puede facilitar un mejor afrontamiento de las demandas habituales de la enseñanza, lo que llevaría a menores niveles de estrés y de burnout docente (Iriarte-Redín y Erro-Garcés, 2020; Mérida-López et al., 2017). Finalmente, dada la vinculación entre la IE y actitudes laborales como la intención de abandono docente (Mérida-López et al., 2020), el desarrollo de estas habilidades podría tener un efecto indirecto sobre los costes del sistema educativo y las bajas laborales.

En España, el profesorado en general, y los docentes en formación inicial en particular, destacan la necesidad de desarrollar las competencias emocionales y sociales de los estudiantes (Montero, 2006). Así, consideran que para ser efectivos en la implementación y el fomento de estas habilidades en clase y en los centros educativos, necesitan una formación inicial en desarrollo emocional y un apoyo de las instituciones desarrollando estrategias formativas para tal fin (Torrijos-Fincias y Martín-Izard, 2014).

Aunque a nivel internacional se han mostrado resultados acerca de la posible mejora de las habilidades de IE del profesorado novel mediante programas de entrenamiento (Vesely et al., 2013), en la actualidad no existen programas sistemáticos dirigidos a mejorar las habilidades emocionales del docente novel. No obstante, son diversos los investigadores que subrayan que el primer paso para desarrollar en los alumnos las competencias emocionales pasa por enseñar a los docentes noveles (Brackett et al., 2009; Schonert-Reichl et al., 2017).

Con ese propósito, se presenta una experiencia formativa piloto con el Programa CRECIENDO (Creando Competencias de Inteligencia Emocional en Nuevos Docentes), dirigido a la formación emocional del profesorado novel, con estudiantes de posgrado que cursan el Máster en Profesorado de Educación Secundaria Obligatoria y Bachillerato, Formación Profesional y Enseñanza de Idiomas de la Universidad de Málaga. Los objetivos específicos fueron: (1) evaluar indicadores de calidad percibida a corto y largo plazo, (2) evaluar posibles diferencias en los niveles de IE antes y después de la aplicación del Programa y (3) examinar las percepciones en cuanto al cumplimiento de expectativas, aprendizajes adquiridos y aspectos positivos y negativos del Programa.

\section{Método}

\section{Participantes}

En la aplicación piloto del Programa participaron 14 estudiantes del Máster, 9 de los cuales asistieron a las dos sesiones de la acción formativa. Los estudiantes fueron 7 hombres y 7 mujeres con un rango de edad de 22 a 51 años $(M=29,07 ; D T=7,90)$ y que habían realizado una media de 25 horas de prácticas $(D T=8,24)$.

\section{Instrumentos}

Questionnaire for Professional Training Evaluation (Q4TE; Grohmann y Kauffeld, 2013). El Q4TE es un cuestionario auto-informado de 12 ítems con una escala de respuesta Likert de 11 opciones de respuestas, con las alternativas que van desde 0 ("completamente en desacuerdo") a 10 ("completamente de acuerdo"). Este cuestionario se compone de seis subdimensiones de satisfacción, tres de evaluación del impacto a corto plazo (i.e., satisfacción, utilidad y conocimiento adquirido) y tres de evaluación del impacto a largo plazo (i.e., aplicación práctica, resultados individuales y resultados organizacionales). Cada subdimensión está compuesta por dos ítems con propiedades psicométricas adecuadas en diferentes contextos (Grohmann y Kauffeld, 2013). 
Wong and Law Emotional Intelligence Scale (WLEIS; Wong y Law, 2002). Para evaluar la IE, se utilizó la adaptación al castellano del instrumento (WLEIS-S; Extremera et al., 2019b). Esta escala de 16 ítems con una escala tipo Likert de 7 puntos evalúa el nivel de IE percibida. Los estudios sobre su estructura factorial han encontrado cuatro factores: percepción intrapersonal (p.ej., "Tengo una buena comprensión de mis propias emociones”), percepción interpersonal (p.ej., "Conozco siempre las emociones de mis amigos a través de sus comportamientos"), asimilación (p.ej., "Soy una persona auto-motivadora") y regulación emocional (p.ej., "Soy capaz de controlar mis propias emociones"). Atendiendo a nuestros objetivos, en los análisis empleamos la puntuación por subescalas y general, con mayores puntuaciones indicando niveles más altos de habilidades emocionales. La versión en español ha mostrado niveles satisfactorios de fiabilidad en estudios con docentes noveles españoles (Mérida-López y Extremera, 2020).

\section{Procedimiento}

Inicialmente, la presente investigación ha seguido un diseño con medidas repetidas pre- y post-intervención con un grupo control comparativo. Desafortunadamente, debido a la escasa participación de docentes noveles en el grupo control, no se pudieron realizar comparaciones entre grupos y sólo se presentan los datos pre- y post-intervención del grupo participante. La aplicación del Programa en formato breve fue formalmente informada y publicitada por los coordinadores del Máster en Profesorado de Educación Secundaria Obligatoria y Bachillerato, Formación Profesio- nal y Enseñanza de Idiomas de la Universidad de Málaga en el curso 2019-20. Se realizó una inscripción online hasta completar el número máximo de participantes para el desarrollo del Programa y se solicitó la participación de los docentes noveles siguiendo las consideraciones éticas nacionales e internaciones. Los investigadores informaron a los potenciales participantes de la voluntariedad y el anonimato de su implicación en el Programa. El procedimiento fue aprobado por el Comité Ético de la Universidad de Málaga (66-2018-H).

\section{Estructura del Programa CRECIENDO}

El Programa CRECIENDO consiste en una intervención de 6 horas de duración, dividida en dos sesiones de 3 horas semanales. Se incluye un primer bloque que recoge información sobre los riesgos psicosociales de la enseñanza, sus causas habituales, emociones negativas y síntomas del estrés docente y consecuencias para el docente novel, aportando herramientas para detectarlo a tiempo y/o prevenirlo, en su caso. Además, se presenta el modelo de habilidad de IE y sus componentes (Mayer et al., 2016): percepción, uso, comprensión y regulación de las emociones (Ver Figura 1). Posteriormente, se desarrolla el papel de la IE y los beneficios para el alumnado y para la salud psicosocial y el trabajo docente. Finalmente, en las siguientes horas del Programa se trabaja sobre cada una de las habilidades de IE ayudando al futuro docente a desarrollar competencias, conocimientos y estrategias para enfrentarse de forma más eficaz al estrés, los conflictos diarios del docente y las problemáticas asociadas (Ver Tabla 1).

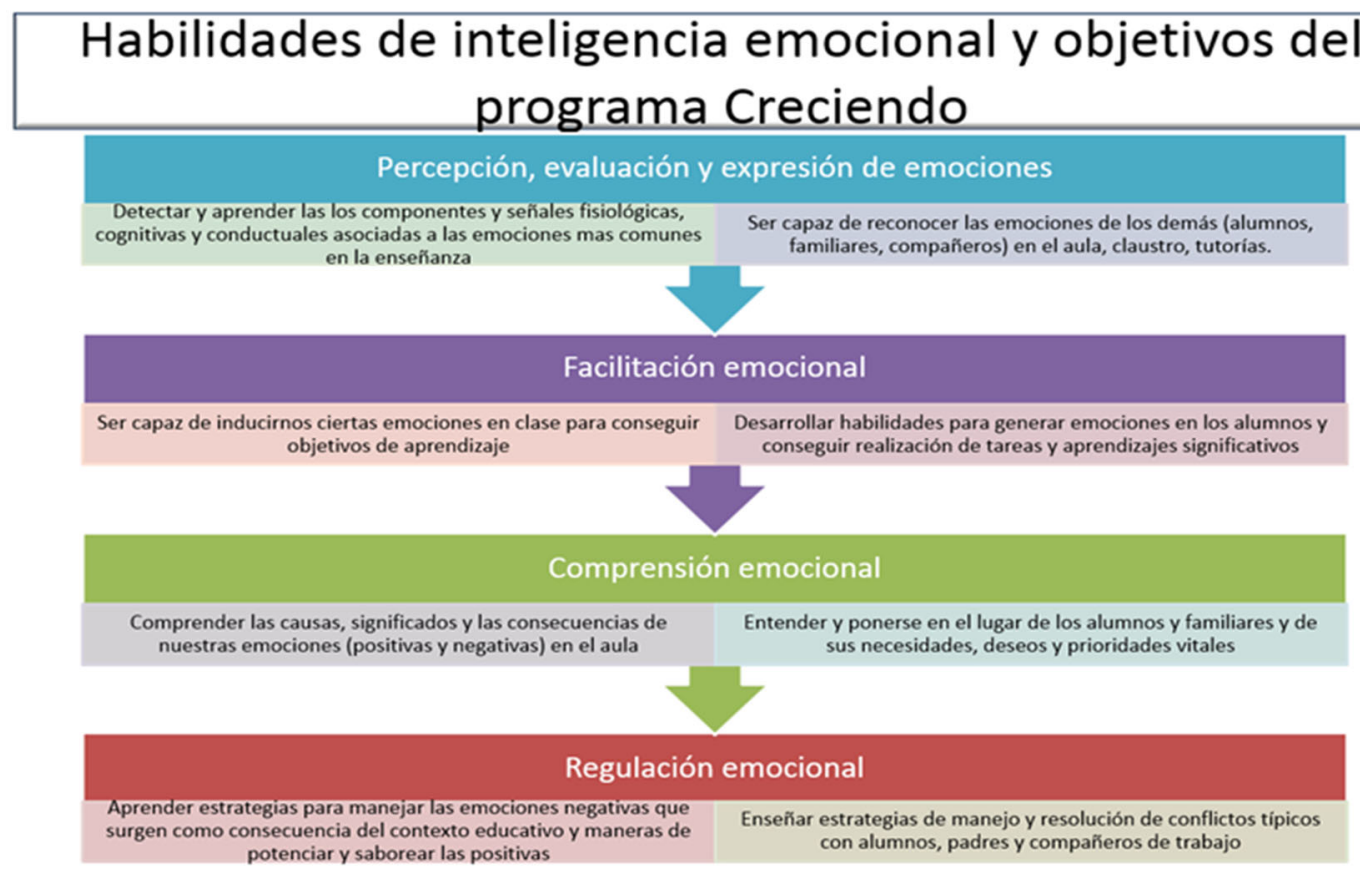

Figura 1.

Habilidades de inteligencia emocional desarrolladas en el Programa CRECIENDO 
Programa “CRECIENDO” (Creando Competencias de Inteligencia Emocional en Nuevos Docentes): Evidencias preliminares y su utilidad percibida en la formación inicial del profesorado de Secundaria

Tabla 1.

Resumen de actividades del Programa CRECIENDO en formato breve (6 horas)

\begin{tabular}{|c|c|c|}
\hline Contenidos & Actividades & Formato(s) \\
\hline $\begin{array}{l}\text { CONTEXTUALIZACIÓN: } \\
\text { DOCENTES DEL SIGLO XXI }\end{array}$ & El desafio de ser docente & $\begin{array}{l}\text {-Psicoeducación (Masterclass) } \\
\text {-Trabajo en equipo } \\
\text {-Recurso bibliográfico }\end{array}$ \\
\hline $\begin{array}{c}\text { PERCEPCIÓN } \\
\text { INTERPERSONAL }\end{array}$ & ¿Emociones propias o ajenas? & $\begin{array}{l}\text { Videoforum y análisis de situa- } \\
\text { ción emocional }\end{array}$ \\
\hline PERCEPCIÓN INTRAPERSONAL & $\begin{array}{c}\text { ¿Qué emociones siento en mi labor docen- } \\
\text { te? } \\
\text { (Actividad de consolidación) }\end{array}$ & $\begin{array}{c}\text { Trabajo individual y exposición } \\
\text { grupal }\end{array}$ \\
\hline FACILITACIÓN & El profesor inducido & Role playing \\
\hline COMPRENSIÓN & Entiendo tu problema & $\begin{array}{l}\text { Role playing de tres docentes } \\
\text { diferentes }\end{array}$ \\
\hline REGULACIÓN & $\begin{array}{l}\text { Herramientas emocionales ante desafios } \\
\text { emocionales ¿Qué hacer ante-durante- } \\
\text { después de surgir la emoción? }\end{array}$ & $\begin{array}{l}\text { Análisis individual de estrategias } \\
\text { regulativas utilizadas }\end{array}$ \\
\hline $\begin{array}{l}\text { INTEGRACIÓN DEL MODELO DE } \\
\text { IE }\end{array}$ & Inteligencia Emocional en acción & Análisis de un caso \\
\hline
\end{tabular}

En cuanto a la metodología del Programa, se trabajan aspectos prácticos con formato de aprendizaje experiencial (p.ej., uso de role playing, escritura reflexiva sobre situaciones, discusión en grupo de conflictos, etc.) basado en su experiencia práctica en el aula (Ver Figura 2). Igualmente, estas actividades se alternan con ejercicios de discusión en parejas ante casos de clase, simulaciones de incidentes crí- ticos (con alumnos, familias o compañeros), o visualización de escenas de vídeo para observar ejemplos de estrategias de regulación emocional efectivas para el manejo de la clase. En todos los casos se ofrece retroalimentación y se lleva a cabo análisis posterior al desarrollo de las actividades.

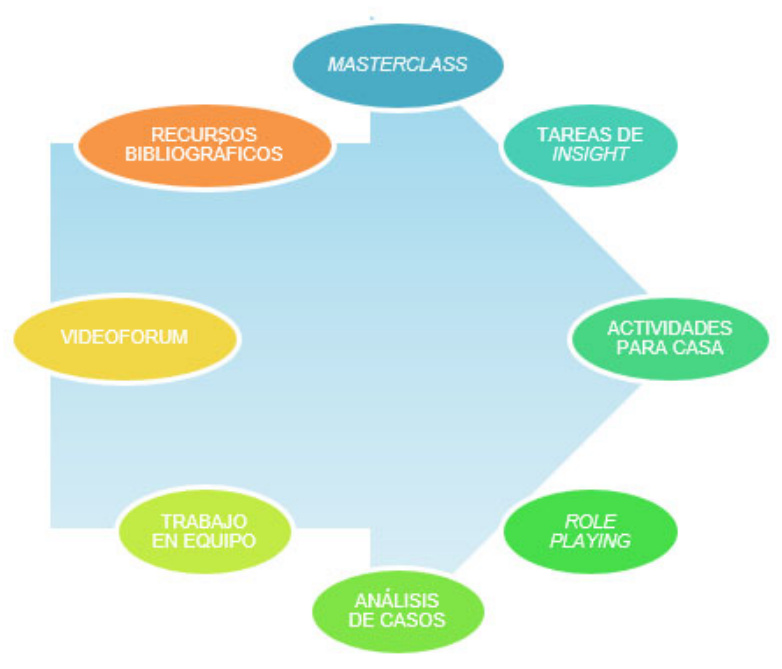

Figura 2.

Ilustración de actividades y tareas de distinto formato desarrolladas en el Programa CRECIENDO 


\section{Resultados}

\section{Análisis descriptivos}

Impacto percibido del Programa CRECIENDO a corto plazo y a largo plazo

Con respecto a evaluación del impacto a corto plazo, en la Tabla 2 se muestran los resultados principales. Tal como se aprecia, las puntuaciones medias en las tres subescalas se encontraron por encima de 7. Se puede resaltar el valor del indicador de utilidad, que fue valorado por los participantes del Programa con 8,43. De esta forma, la gran mayoría de los participantes consideraron que el Programa CRECIENDO podría ser beneficioso para las prácticas docentes $\mathrm{y}$, además, afirmaron que utilizarían el conocimiento que han obtenido en la formación en su carrera profesional. Con respecto a la evaluación del impacto a largo plazo, la Tabla 2 muestra que las medias en las tres subescalas estuvieron de nuevo por encima de 7 puntos. Se puede resaltar el indicador de aplicabilidad, que fue valorado por los participantes del Programa con 8,12 puntos. Así, la mayor parte de los participantes afirmaron que la aplicación de los contenidos de la formación a través del Programa CRECIENDO facilitará en el futuro su competencia docente y que su desempeño mejorará gracias a los contenidos aprendidos en el Programa.

Tabla 2.

Indicadores de evaluación del impacto a corto plazo y a largo plazo de la aplicación del Programa CRECIENDO.

\begin{tabular}{lcc}
\hline Variables & Media & Desviación Típica \\
\hline Indicadores a corto plazo & 7,62 & 1,09 \\
1. Satisfacción & 8,43 & 1,49 \\
2. Utilidad & 7,31 & 2,44 \\
3. Conocimiento & & \\
Indicadores a largo plazo & 8,12 & 1,02 \\
1. Aplicabilidad & 7,06 & 2,54 \\
2. Resultados individuales & 7,56 & 1,59 \\
3. Resultados organizacionales & & \\
\hline
\end{tabular}

\section{Análisis de diferencias de medias pre- y post- intervención en las habilidades de inteligencia emocio- nal}

Para determinar el posible efecto del Programa en los niveles de las distintas habilidades emocionales, se realizaron análisis descriptivos (medias y desviaciones típicas) y de varianza ( $t$ de Student para muestras relacionadas) con cada una de las puntuaciones obtenidas en los instrumentos comparando las puntuaciones pre-intervención (T1) con las puntuaciones post-intervención (T2) en el grupo de docentes noveles que participaron en el Programa CRECIENDO.

Tal como se aprecia en la Tabla 3, a pesar de la muestra reducida, se observan tendencias estadísticas en las puntuaciones de la dimensión de percepción interpersonal $\left(M_{\text {pre- }}\right.$ intervención $\left.=5,55 ; M_{\text {post-intervención }}=6,16 ; p=0,07\right)$, encontrándose un aumento en dichas puntuaciones en la evaluación post-intervención. Se encontraron diferencias estadísticamente significativas en la dimensión de asimilación emocional $\left(M_{\text {pre-intervención }}=5,08 ; M_{\text {post-intervención }}=5,72 ; p<0,01\right)$, con un incremento significativo en esta habilidad al concluir el Programa. Finalmente, se obtuvieron diferencias significativas en la puntuación total de IE $\left(M_{\text {pre-intervención }}=\right.$ 5,29; $\left.M_{\text {post-intervención }}=5,70 ; p=0,01\right)$. Concretamente, al finalizar el Programa CRECIENDO, el grupo de profesores noveles asistentes informaron de mayores puntuaciones en su IE general que al inicio del Programa. 
Programa “CRECIENDO” (Creando Competencias de Inteligencia Emocional en Nuevos Docentes): Evidencias preliminares y su utilidad percibida en la formación inicial del profesorado de Secundaria

Tabla 3.

Estadísticos descriptivos pre- y post-intervención de las habilidades de inteligencia emocional

\begin{tabular}{|c|c|c|c|c|c|c|}
\hline \multirow[b]{2}{*}{ Variables } & \multicolumn{2}{|c|}{ Pre-intervención } & \multicolumn{2}{|c|}{ Post-intervención } & \multirow[b]{2}{*}{$t$} & \multirow[b]{2}{*}{$(g l)$} \\
\hline & $M$ & $D T$ & $M$ & $D T$ & & \\
\hline Percepción intrapersonal & 5,33 & 1,13 & 5,50 & 1,24 & $-1,51$ & (8) \\
\hline Percepción interpersonal & 5,55 & 0,90 & 6,16 & 0,81 & $-2,03$ & $(8)^{+}$ \\
\hline Asimilación & 5,08 & 0,81 & 5,72 & 0,93 & $-3,41$ & $(8)^{* *}$ \\
\hline Regulación & 5,22 & 1,07 & 5,41 & 1,25 & $-0,88$ & (8) \\
\hline IE global & 5,29 & 0,80 & 5,70 & 0,93 & $-3,32$ & $(8)^{*}$ \\
\hline
\end{tabular}

Nota: $\mathrm{IE}=$ Inteligencia Emocional; $g l=\operatorname{grados}$ de libertad $;{ }^{+} p \leq 0,10 ;{ }^{*} p \leq 0,05 ;{ }^{* *} p \leq 0,01$.

\section{Análisis de las expectativas cumplidas, utilidad percibi- da y aspectos positivos y negativos del Programa}

Al final de la segunda sesión del Programa se preguntó sobre la utilidad percibida del Programa, así como los aprendizajes adquiridos y los pros y contras de su realización, con una serie de preguntas abiertas.

Con respecto a la pregunta referida a las expectativas, la mayoría de los docentes noveles informaron de que sus expectativas habían sido cubiertas parcialmente. Una de las razones expuestas es que el Programa había sido breve en su duración y no se pudo profundizar en el conocimiento sobre estrategias para casos e incidencias en el aula concretos. Por ello, aunque se ha proporcionado información sobre estrategias, se ha subrayado la necesidad de un seguimiento personal y de profundizar en el conocimiento procedimental, es decir, de proporcionar pautas sobre cómo aplicar estas herramientas en situaciones de manejo emocional específicas del aula. Algunos ejemplos de las respuestas dadas por los participantes han sido:

"Sí, he aprendido algunas técnicas que era lo que esperaba”.

"Me habría gustado recibir más información especifica, como técnicas para enfrentarnos a situaciones dificiles. Me explico; me habría gustado que se plantearan problemas reales con situaciones reales. P.ej,.: Niño disruptivo, técnicas para reubicar la clase”.

"En parte sí, porque he conocido conceptos y perspectivas diferentes, pero en mi opinión, ha sido muy breve $y$, en general, se han visto pinceladas de algo que considero que es esencial tanto para docentes como para otras personas pertenecientes a cualquier profesión”.

"Sí y no. He aprendido mucho con respecto a la gestión de emociones, pero pienso que para determinados aspectos necesitaría un seguimiento personal”.

"Parcialmente. Lo esperaba algo más práctico. Aunque la información ha sido muy útil y los ponentes, expertos y agradables".

"Sí, era expositivo sin ir a contenido muy concreto".

"Lo cierto es que quería saber cómo gestionar mejor mis emociones y sobre todo mi inseguridad. Creo que después de estos dos talleres, con todos esos casos que se nos han mostrado de estudios profesionales de la realidad que viven los docentes hoy día y cómo nos han enseñado a saber cuáles son esas emociones clave, por qué son causadas y el proceso para controlarlas ha cumplido mis expectativas, además de las actividades hechas en los talleres".

En relación al aprendizaje que han adquirido durante el Programa, los docentes en formación inicial han subrayado la adquisición de diversas técnicas y estrategias para manejar y controlar sus emociones y la de sus alumnos en ciertos momentos. La conciencia de sus emociones, la importancia de su estado de ánimo y el de sus alumnos y la necesidad de percibirlos, comprenderlos y gestionarlos han sido tópi$\cos$ frecuentemente manifestados en sus respuestas. Algunos ejemplos de las respuestas proporcionadas por los participantes han sido:

"Algunas técnicas para regular las emociones o anticiparme a ellas".

"Reconocer, visibilizar y gestionar sentimientos". 
“Algunas estrategias para intentar controlar las emociones”.

“Comprensión y regulación de emociones. Estrategias”.

"Sobre todo, que hay problemas mucho peores y que no estoy sola, es decir, que la mayoría de situaciones ya les han pasado a otras personas y debemos aprender de ello”.

“A la importancia de mi estado de ánimo en el alumnado. A percibir mayor rango de comportamientos emocionales".

"Que como futuros docentes somos espejos para nuestros alumnos, nuestras emociones se pueden percibir y hemos de aprender a saber gestionarlas. Pero no solo eso, aprender a percibirlas en el alumnado nos ayudará a mejorar la convivencia, la enseñanza y la atención que necesitan como alumnos y seres humanos que son. Me ha ayudado a saber que emociones hay de modo más específico, por qué son causadas y los medios para saber guiarles".

En cuanto a los aspectos positivos (pros) y negativos (contras) informados por los participantes sobre el Programa, la gran mayoría ha valorado positivamente los consejos, los ejemplos empleados y la cercanía de los docentes tratando de generar un clima emocional colaborativo que fomentase la participación y la confianza del grupo. Algunos ejemplos de las respuestas dadas por los docentes en formación inicial han sido:

“Los ejemplos prácticos y las dinámicas”.

“La profesionalidad y el sentido del humor del equipo”.

"La cercanía de los docentes. La variedad de información. La amplitud de miras".

“Los consejos que me llevo”.

"La forma en la que se ha impartido, de manera cercana y en un ambiente de confianza".

“Las prácticas en el aula”.

"Me gustó el modelo que nos enseñaron para trabajar las emociones, nunca había caído en esa manera de actuar ante una situación emocional dificil: identificar el sentimiento, utilizarlo, determinar su causa y llevarlo a la acción enfocándolo desde el punto de vista de qué emoción querría sentir. La verdad es que el primer taller, la actividad del profesor inducido que hicieron los compañeros como docentes y nosotros como alumnos hipotéticos fue un ejercicio muy útil para ver esas situaciones y figurarnos cómo actuar. Ambas cosas me gustaron además de la actividad en conjunto del primer taller de la plantilla de compromiso emocional docente, me ayudó compartir y ver las otras ideas de los compañeros".

En relación a los aspectos más negativos, la mayoría de los participantes subrayó la brevedad del Programa y la necesidad de más interacción. Además, manifestaron que el enfoque psicoeducativo utilizado para explicar las herramientas no permitía, por falta de tiempo, ponerlas todas en práctica con casos reales y con ejemplos durante las sesiones. Ejemplos de las respuestas dadas por los docentes en formación inicial han sido:

"Que la participación activa de los asistentes ha sido poco incentivada”.

“El poco tiempo que ha durado”.

"La brevedad".

"Me han faltado más prácticas, sobre todo, ligadas al estrés y a la ansiedad".

"Las explicaciones un tanto largas, que no siempre se entiende a la primera”.

Por último, se preguntó a los docentes noveles si recomendaría el Programa a otros compañeros del Máster en Profesorado de Secundaria que no habían participado, con objeto de continuar ofertando este Programa en el Máster. Todos, sin excepción, valoraron la necesidad de incorporar esta formación dentro de los contenidos del Máster y, además, manifestaron la necesidad de su realización por otros compañeros para incorporar las herramientas de gestión emocional en el aula dada su escasa experiencia en el manejo de situaciones conflictivas en el aula. Ejemplos de las respuestas dadas por los docentes en formación inicial han sido:

"Sí, Por la necesidad en el ámbito educativo de la adquisición y el manejo de la inteligencia emocional”.

"Sí, porque considero que son muy importantes las habilidades emocionales para la práctica docente”.

"Sí, porque viene muy bien para gestionar las emociones en clase”.

"Sí, porque es muy interesante y puede resultar útil, además de aumentar los conocimientos sobre el tema”.

"Desde luego. Más que nada por la necesidad de consciencia y las estrategias que podemos sacar no sólo del taller, sino de los compañeros también”.

"Sí, la mayoría de mis compañeros/as lo requiere”.

"Para muchos de nosotros, por no decir la mayoría, es nuestra primera vez como docentes, se nos conciencia de la necesidad de adaptarnos a esta era de la información, tecnología e innovación y somos conscientes del estrés y trabajo que nos generará y si hay algo que nos acompaña cada día como seres humanos son nuestras emociones naturales. Y son muchos los nervios y necesidades que tenemos para saber gestionarlas delante de nuestros alumnos $y$ 
saber ayudarles con las suyas para que así seamos maestros de humanidad, como debe ser. Por ello, recomendaría este taller a cualquiera de mis compañeros".

En definitiva, las respuestas obtenidas parecen sugerir que la totalidad de los asistentes recomendarían este Programa al resto de compañeros del Máster en Profesorado de Secundaria. Además, el Programa es percibido como una formación necesaria que contribuye a un mejor bienestar personal y laboral como docente, que repercutirá también en la calidad de las relaciones profesor-alumno en clase y en la forma de afrontar los constantes desafíos, incidentes y dificultades que se encontrará el profesorado a lo largo de su labor pedagógica.

\section{Discusión}

El objetivo de esta investigación ha sido examinar el posible impacto a corto y a largo plazo de la implementación de un Programa breve de IE aplicado a futuros docentes en su formación inicial en el Máster en Profesorado de Secundaria de la Universidad de Málaga. Igualmente, de forma muy preliminar se examinó si los niveles de IE, tras la implementación del Programa, se incrementaban en aquellos docentes noveles que participaron en el Programa. Por último, se evalúo la percepción del cumplimiento de expectativas, de aprendizajes adquiridos y los aspectos positivos y negativos percibidos durante la impartición del Programa.

En líneas generales, los hallazgos muestran que los docentes noveles consideran de gran utilidad, a corto plazo, y de gran aplicabilidad en su carrera profesional a largo plazo, el aprendizaje y la enseñanza de herramientas socioemocionales que les ayuden a afrontar los estresores docentes cotidianos. La literatura ha confirmado que parte de los niveles elevados de estrés docente en los profesores noveles podría derivarse de un desajuste formativo en recursos y estrategias de desarrollo socio-emocional (Meyer, 2009; Schutz y Zembylas, 2009; Stuart y Thurlow, 2000). Estas deficiencias estarían afectando negativamente en el bienestar del docente e, indirectamente, afectarían a su rendimiento laboral y, consecuentemente, al aprendizaje recibido por los alumnos en su materia (Braun et al., 2020; Jennings, y Greenberg, 2009).

Los hallazgos de este estudio preliminar muestran algunas mejoras estadísticamente significativas entre el profesorado novel que participa en el Programa CRECIENDO comparado con sus puntuaciones al inicio. Los efectos del Programa CRECIENDO en este estudio piloto pueden resumirse en: (1) tendencia estadística de mejora en la dimensión percepción interpersonal, (2) mejora significativa en la dimensión asimilación emocional y (3) aumento significativo en la puntuación global de IE de los profesores noveles participantes en el Programa. Sin embargo, en cuanto a las dimensiones percepción interpersonal y regulación emocio- nal, aunque las puntuaciones fueron mayores al finalizar el Programa, no se confirmaron aumentos estadísticamente significativos.

De forma general, nuestros resultados evidencian un apoyo parcial a las mejoras en las habilidades de IE entre el profesorado que participó en esta experiencia piloto de aplicación del Programa CRECIENDO. Estos hallazgos van en línea con los encontrados en otras investigaciones con profesorado novel que demuestran la eficacia de las intervenciones en la mejora de la IE y muestran incrementos en habilidades emocionales auto-informadas de los docentes participantes (Vesely et al., 2013). Igualmente, la falta de aumento significativo en las puntuaciones en asimilación y regulación podría explicarse, en parte, por la reducción de poder estadístico debido al pequeño tamaño muestral en relación con la participación en las dos sesiones del Programa CRECIENDO. Además, es posible que una intervención más extensa en contenidos y sesiones o prolongada en el tiempo pudiera incrementar significativamente los resultados de mejora en las habilidades emocionales. No obstante, estos resultados suponen un punto de partida para identificar y dirigir nuestros esfuerzos hacia el desarrollo de recursos y herramientas que ayudan a mejorar el bienestar docente $\mathrm{y}$, de forma indirecta, las interacciones en el aula y los resultados académicos del alumnado (Jennings y Greenberg, 2009). Las habilidades emocionales se valoran como un recurso necesario en el entrenamiento de los profesores noveles según su percepción de utilidad y aplicabilidad en el aula como futuros docentes. Así, el constructo de IE podría ser un marco de referencia que permita elaborar modelos de desarrollo competenciales docentes y Programas de entrenamiento más efectivos para tratar los conflictos en el aula (Vesely-Maillefer y Saklofske, 2018).

En cuanto a los análisis de las expectativas, aprendizajes y aspectos positivos y negativos del Programa, la mayoría de los participantes informaron de que sus expectativas han sido cubiertas parcialmente debido a la breve duración del curso y a la imposibilidad de profundizar en casos concretos, subrayando la necesidad de un seguimiento personal. Futuras adaptaciones de este Programa deberán estudiar la necesidad de aumentar su duración y la inclusión de actividades de aplicación específica. Con respecto al aprendizaje, los docentes noveles han subrayado la adquisición de diversas técnicas y estrategias para manejar y controlar sus emociones y la de sus alumnos en ciertos momentos. La conciencia de sus emociones, la importancia de su estado de ánimo y el de sus alumnos y la necesidad de percibir, comprender y gestionarlos han sido tópicos claves que han salido en sus respuestas. Así, consideran que estos recursos y herramientas afectivo-emocional son parte esencial de su repertorio competencial como docentes.

En relación a los aspectos positivos del Programa, la gran 
mayoría de los participantes ha valorado positivamente los consejos, ejemplos y cercanía de los docentes tratando de generar un clima emocional colaborativo que fomentase la participación y confianza del grupo. En cuanto a los aspectos más negativos, la mayoría subrayó la brevedad, la necesidad de más interacción y mayor enfoque práctico con casos reales y ejemplos durante la clase. Esta retroalimentación permitirá seguir fortaleciendo el carácter práctico y aplicado a lo largo del Programa, sin perder la necesidad de una contextualización y explicación psicoeducativa de la utilidad y forma correcta de utilizar cada recurso emocional. Finalmente, con respecto a la utilidad percibida del Programa, se observa en que todos los asistentes del taller recomendarían este Programa al resto de compañeros del Máster en Profesorado de Secundaria que no participaron en él. Nuestro objetivo será trabajar en las mejoras para su incorporación en las futuras ediciones del Máster como parte de la formación de las nuevas generaciones de docentes (Vesely-Maillefer y Saklofske, 2018).

Por último, este estudio piloto presenta bastantes limitaciones que conviene señalar. En primer lugar, los datos se han obtenido con un grupo reducido de docentes noveles de una edición del Máster en Profesorado de Secundaria de la Universidad de Málaga, por lo que las dificultades de generalización de estos hallazgos son evidentes. Además, nuestros datos deben tomarse con mucha cautela y de modo preliminar, dado que no fue posible obtener un grupo control, necesario para demostrar las mejoras significativas con diseños cuasi-experimentales. Próximas investigaciones deberán realizarse con un grupo de control activo (que realiza otro tipo de formación no vinculada con el desarrollo de competencias emocionales; Kotsou et al., 2019). Igualmente, será necesario incluir otros indicadores de IE, tales como las medidas de habilidad y de juicio situacional e incluir actitudes hacia la enseñanza (p.ej., satisfacción laboral, engagement...). Por último, este estudio no permitió realizar una evaluación de seguimiento a lo largo del curso. Futuros trabajos con módulos de desarrollo de competencias socio-emocionales instaurados dentro de los Másteres en Profesorado de Secundaria deberían evaluar si los resultados se mantienen o pierden intensidad con el paso del tiempo, lo cual nos ayudaría a diseñar intervenciones con sesiones de seguimiento personal a lo largo de proceso de desarrollo formativo y competencial, por ejemplo, durante su periodo de prácticas.

En conclusión, aunque es necesario la generalización de nuestros resultados con enfoques cuasi-experimentales con grupo control, muestras más amplias y evaluaciones a largo plazo, nuestros hallazgos parecen sugerir que los docentes noveles valoran positivamente la formación inicial en habilidades emocionales aplicadas al aula y que, incluso con intervenciones breves de sólo 6 horas de duración, se observan algunas mejoras significativas en ciertas habilidades emocionales claves de la IE y una percepción de utilidad del Programa en los docentes noveles. Si se confirman estos resultados en próximos estudios, se apoyaría la idoneidad de integrar asignaturas o Programas más extensos y aplicados de formación emocional docente en los Másteres en Profesorado de Educación Secundaria como herramienta para alcanzar un mejor bienestar docente y, por ende, un clima más positivo en clase que redunde en mejores resultados académicos, emocionales y sociales en los alumnos.

\section{Financiación}

Esta investigación ha sido financiada por el Proyectos $\mathrm{I}+\mathrm{D}+\mathrm{i}$ en el marco del Programa Operativo FEDER Andalucía 2014-2020 (UMA18-FEDERJA-147) y por el Ministerio de Educación $\mathrm{y}$ Formación Profesional (FPU16/02238).

\section{Referencias}

Alvarez, H.K. (2007). The impact of teacher preparation on responses to student aggression in the classroom. Teaching and Teacher Education, 23(7), 11131126. https://doi.org/10.1016/j.tate.2006.10.001

Brackett, M.A., Patti, J., Stern, R., Rivers, S.E., Elbertson, N.A., Chisholm, C. y Salovey, P. (2009). A Sustainable, Skill-Based Approach to Building Emotionally Literate Schools. En M. Hughes, H.L. Thompson, y J.B. Terrell (Eds.), Handbook for Developing Emotional and Social Intelligence: Best Practices, Case Studies, and Strategies (pp. 329-358). Pfeiffer/John Wiley \& Sons.

Braun, S.S., Schonert-Reichl, K.A. y Roeser, R.W. (2020). Effects of teachers' emotion regulation, burnout, and life satisfaction on student well-being. Journal of Applied Developmental Psychology, 69, 101151. doi:10.1016/j.appdev.2020.101151

Extremera, N., Mérida-López, S. y Sánchez-Gómez, M. (2019a). La importancia de la inteligencia emocional del profesorado en la misión educativa: impacto en el aula y recomendaciones de buenas prácticas para su entrenamiento. Voces de La Educación, 4 (9), 74-97.

Extremera, N., Rey, L. y Sánchez-Álvarez, N. (2019b). Validation of the Spanish version of Wong Law Emotional Intelligence Scale (WLEIS-S). Psicothema, 31(1), 94-100. http://dx.doi.org/10.7334/ psicothema2018.147

Grohmann, A. y Kauffeld, S. (2013). Evaluating training programs: Development and correlates of the Questionnaire for Professional Training Evaluation. International Journal of Training and Development, 17(2), 135-155. doi:10.1111/ijtd.12005 
Programa “CRECIENDO” (Creando Competencias de Inteligencia Emocional en Nuevos Docentes): Evidencias preliminares y su utilidad percibida en la formación inicial del profesorado de Secundaria

Iriarte-Redín, C. y Erro-Garcés, A. (2020). Stress in teaching professionals across Europe. International Journal of Educational Research, 103, 101623. https://doi.org/10.1016/j.ijer.2020.101623

Jennings, P.A. y Greenberg, M.T. (2009). The prosocial classroom: Teacher social and emotional competence in relation to student and classroom outcomes. Review of Educational Research, 79(1), 491-525. https://doi.org/10.3102/0034654308325693

Jennings, P.A., Snowberg, K.E., Coccia, M.A. y Greenberg, M.T. (2011). Improving classroom learning environments by cultivating awareness and resilience in education (CARE): Results of a randomized controlled trial. Journal of Classroom Interaction, 46(1), 37-48.

Kotsou, I., Mikolajczak, M., Heeren, A., Grégoire, J. y Leys, C. (2019). Improving emotional intelligence: A systematic review of existing work and future challenges. Emotion Review, 11(2), 151-165. https://doi.org/10.1177/1754073917735902

Mayer, J.D., Caruso, D.R. y Salovey, P. (2016). The ability model of emotional intelligence: Principles and updates. Emotion Review, 8(4), 290-300. doi:10.1177/1754073916639667

Mérida-López, S. y Extremera, N. (2017). Emotional intelligence and teacher burnout: A systematic review. International Journal of Educational Research, 85, 121-130. doi:10.1016/j.ijer.2017.07.006

Mérida-López, S. y Extremera, N. (2020). Cuando la falta de compromiso ocupacional del profesorado novel no es suficiente para explicar la intención de abandono: ¡la inteligencia emocional importa! Revista de Psicodidáctica, 25(1), 52-58. https:// doi.org/10.1016/j.psicod.2019.05.001

Mérida-López, S., Sánchez-Gómez, M. y Extremera, N. (2020). Leaving the teaching profession: Examining the role of social support, engagement and emotional intelligence in teachers' intentions to quit. Psychosocial Intervention, 29(3), 141-151. https:// doi.org/10.5093/pi2020a10

Meyer, D.K. (2009). Entering the Emotional Practices of Teaching. En P. Schutz y M. Zembylas (Eds.), Advances in Teacher Emotion Research: The Impact on Teachers' Lives (pp. 73-91). Springer.

Montero, L. (2006). Profesores y profesoras en un mundo cambiante: el papel clave de la formación inicial. Revista de educación, 340(1), 66-85.

Oberle, E. y Schonert-Reichl, K.A. (2016). Stress contagion in the classroom? The link between classroom teacher burnout and morning cortisol in elementary school students. Social Science and Medicine, 159, 30-37. https://doi.org/10.1016/ j.socscimed.2016.04.031

Oberle, E. y Schonert-Reichl, K.A. (2017). Social and Emotional Learning: Recent Research and Practical Strategies for Promoting Children's Social and Emotional Competence in Schools. En J.L. Matson (Ed.), Handbook of Social Behavior and Skills in Children, Autism and Child Psychopathology Series (pp. 175-197). Springer International Publishing.

Schonert-Reichl, K.A., Kitil, M.J. y Hanson-Peterson, J. (2017). To reach the students, teach the teachers: $A$ national scan of teacher preparation and social and emotional learning. A report prepared for the Collaborative for Academic, Social, and Emotional Learning (CASEL). Vancouver: University of British Columbia.

Schutz, P. y Zembylas, M. (Eds.). (2009). Advances in Teacher Emotion Research: The Impact on Teachers' Lives. Springer.

Stuart, C. y Thurlow, D. (2000). Making it their own: Preservice teachers' experiences, beliefs, and classroom practices. Journal of teacher education, 51(2), 113121. https://doi.org/10.1177\% 2F002248710005100205

Torrijos-Fincias, P. y Martín-Izard, J.F. (2014). Desarrollo de competencias emocionales en el profesorado de educación secundaria a través de una intervención por programas. Teoría de la Educación. Educación y Cultura en la Sociedad de la Información, 15(1), 90-105.

Vesely, A.K., Saklofske, D.H. y Nordstokke, D.W. (2014). EI training and pre-service teacher wellbeing. Personality and Individual Differences, 65, 81-85. https://doi.org/10.1016/j.paid.2014.01.052

Vesely-Maillefer, A.K. y Saklofske, D.H. (2018). Emotional Intelligence and the Next Generation of Teachers. En K.V. Keefer, J.D.A. Parker y D.H. Saklofske (Eds.), Emotional Intelligence in Education: Integrating Research with Practice (pp. 377402). Springer.

Wong, C.S. y Law, K.S. (2002). The effects of leader and follower emotional intelligence on performance and attitude: An exploratory study. Leadership Quarterly, 13, 243-274. https://doi.org/10.1016/ S10489843(02)00099-1 